\title{
Rinsing with chlorhexidine may reduce incidence of dry socket after third molar surgery
}

\author{
When extraction of mandibular third molars is required, does use of a \\ chlorhexidine rinse reduce the incidence of dry socket (alveolar osteitis)?
}

\section{Caso A, Hung LK, Beirne OR. Prevention of alveolar osteitis with chlorhexidine: a meta-analytic review. Oral Surg Oral Med Oral Pathol Oral Radiol Endod 2005; 99:155-159}

Data sources Medline and the Science Citation Index were used and searches were made by hand for studies published in any language.

Study selection Studies included were human clinical trials evaluating the effect of a chlorhexidine (CHX) rinse on the incidence of alveolar osteitis (AO), following extraction of mandibular third molars, if they randomly assigned individuals to control and $\mathrm{CHX}$ rinse groups that used similar criteria to diagnose AO.

Data extraction and synthesis Studies were screened by three examiners independently. Due to protocol differences, studies were separated into two groups. Group 1 included studies that evaluated subjects who rinsed with $\mathrm{CHX}$ only on the day of surgery and Group 2 studies that evaluated patients who used multiple rinses with $\mathrm{CHX}$.

Results The relative risk for the single-rinse group was $1.36(95 \%$ confidence interval $(\mathrm{Cl}), 0.80-2.33 ; P>0.05)$, whereas for the multiplerinse group, relative risk was $1.90(95 \% \mathrm{Cl}, 1.46-2.47, P<0.05)$.

Conclusions It was not possible to determine if a single rinse with $\mathrm{CHX}$ on the day of surgery significantly reduced the incidence of $\mathrm{AO}$. The results of this study do, however, support the conclusion that rinsing at least on the day of surgery and for several days following the removal of the teeth reduces the incidence of $A O$ associated with the extraction of mandibular third molars. The minimum number of days of rinsing needed to produce this effect could not be determined by this analysis. Further studies using a standard definition for $\mathrm{AO}$, and stratification of patients by gender, difficulty of extraction, smoking etc., are needed to determine the best use for $\mathrm{CHX}$ rinses in subjects having the mandibular third molar removed.

\section{Commentary}

This meta-analysis conforms with published standards and relates to a frequent complication after one of the most frequently performed surgical operations world-wide. Careful analysis has addressed such issues as publication bias (none was detected). As so often, problems were encountered with the constituent randomised trials: in this meta-analysis the problems were inconsistent recording, lack of specificity with regard to the definition of dry socket and confusion over the unit of assessment (patient or operative site). This meta-analysis gives a clear answer, however, that repeat rinses with CHX starting on the day of surgery reduced the incidence of dry socket, whereas a single rinse on the day of surgery did not. The RR strongly suggest that this result is significant clinically as well as statistically, particularly given the high frequency of this particular complication.

This meta-analysis also helps to set the agenda for future research: one question that follows is whether the costs and complications of repeated $\mathrm{CHX}$ rinses justifies routine use. Most importantly, perhaps, this meta-analysis sheds light on the causal relationship between oral microflora and AO. Lack of significant risk reduction associated with a CHX rinse only on the day of surgery suggests that infection control and microflora population reduction on the day of operation is less important than in subsequent days, when oral hygiene maintenance is of course more difficult.

These data should not be used to justify prophylactic third molar removal on the grounds that post-operative infection is more likely if there is a recent history of, or current pericoronitis, since predicting the onset of pericoronitis with the necessary precision is not possible. Nevertheless, this meta-analysis is a particularly welcome contribution in the modern era, in which surgical intervention is reserved for those people who develop third molar-related disease.

\section{Practice point}

- This meta-analysis provides clinically significant evidence that repeated CHX mouth rinses, beginning on the day of third molar removal, reduce the incidence of dry socket but single rinses on the day of surgery do not.

\author{
Jonathan Shepherd \\ Department of Oral and Maxillofacial Surgery, University of \\ Wales College of Medicine, University of Cardiff, Cardiff, \\ Wales, UK
}

Evidence-Based Dentistry (2005) 6, 36. doi: $10.1038 /$ sj.ebd. 6400333 